\title{
Research Review
}

\section{Heather McLellan}

\section{Citation}

Punches, B. E., Berger, K. M., Freiermuth, C. E., Soliman, S. A., Walker, Q. T., \& Lyons, M. S. (2021). Emergency nurse perceptions of pain and opioids in the emergency department. Pain Management Nursing. https://doi.org/10.1016/j.pmn.2021.05.003

\section{Background}

$\mathrm{T}$ he authors for this study identify that opioid use disorder and overdose-related death statistics are on the rise and have reached crisis proportion. The authors note that for many patients, the emergency department (ED) is the first exposure to opioids when used for pain management. Understanding emergency nurse knowledge, attitudes and behaviours will have a direct impact on both pain management and opioid use, the authors sought to have a better understanding of the perspective of the emergency nurse.

\section{Purpose of the study}

The purpose of the study is to identify emergency nurses' knowledge and attitudes towards ED pain management in the context of the ongoing opioid use crisis.

\section{Research approach and methods}

The authors used a qualitative exploratory approach. Researchers used a demographic survey to understand the study participants' background then engaged in semi-structured interviews with open-ended questions to collect their data. Interviews were conducted one-on-one and completed by a single researcher for increased consistency. Interview questions focused on emergency care including pain management and opioid use disorder (OUD). Transcripts of the interviews were reviewed, coded and conventional content analysis done independently, as well as jointly, by the researchers.

\section{Setting and sample}

This study uses a convenience sample of emergency nurses at a single urban Level 1 trauma centre. Participants were recruited using email, word of mouth and flyers in the department. Interested individuals then contacted the researchers to participate. Included were nurses having at least 12 months of emergency department nursing experience at the centre. Excluded were those who were serving in management roles. The rationale for this selection is they wanted nurses who were actively caring for patients.

\section{Findings}

The authors had a response rate of 12 nurses. One component of the research was the collection of demographic information from participants, but that information articulated in the article. The authors identified two primary themes: "Nurses Influence ED Pain Management" and "Adjustments in ED pain management" under a predominant concept that "Pain Management Depends on the Care Team". The domains emerging under the "Nurses Influence ED Pain Management" theme included subjective versus objective assessment of pain and response to pain requests. Domains under "Adjustments in ED pain management" were the potential facilitators and barriers to pain management.

\section{Commentary}

This study identifies the influence that nurses have over pain management. The authors determined that patient behaviours often influenced both the subjective and objective assessment of pain, particularly when the patient was angry or defensive. This is consistent with the findings of similar studies. Sardo et al (2020) reported that while frequently assessed at triage, pain is less-well assessed at the bedside leading to suboptimal pain management. Moceri and Drevdahl (2014) also found patient behaviours influence nurses' assessment and choices in pain management, so that they often used their own assessment of the patient's pain instead of basing pain management on the description provided by the patient. Abouzida et al. (2020) found screening for pain at the bedside occurred with just over half of the patients, in addition to pain scale used in only $10 \%$ of cases.

The authors address both facilitators and barriers to pain management. Concern about OUD and addiction issues or protocolized pain management strategies are potential barriers to effective pain management. Other authors have also identified these barriers. Abouzida et al. (2020) note that although pain undeniably has negative consequences, pain management often goes under addressed. Moceri and Drevdahl note nurses under treatment of pain often focused on concerns around future addiction and dependence around opioid use, which is consistent with the findings in this study. Addiction and condoning drug seeking behaviour/opioid use was also a key theme from Punches et al. (2020). 
Facilitators to pain management identified in the study included education and discussion around treatment alternatives and expectations. Kahsay and Pitkajarvi (2019) also identified the use of training modules for nurses addressing both knowledge and attitudes, allowed for better assessment of patient and improved pain management overall.

One primary strengths of this study is the timeliness of the message. The World Health Organization (2021) identified OUD as affecting 36.3 million people around the world in 2019. Emergency nurses work in a landscape where OUD is an increasing crisis of global proportions. A second strength of the study is the choice of one-on-one interviews allowing the interviewer to provide a safe space for discussion and hear the key messages from each participant without distraction.

A recommendation to strengthen this paper would be to include a better understanding of the participant's level of experience and the study location, particularly around patient demographics. The authors also identify the limitations of using a single site for their data collection. A larger and more diverse study population would strengthen the research study.

\section{Key messages}

- Nurses have significant impact on pain management strategies in the ED.

- Concern about addiction and OUD can negatively influence pain management.

- Emergency nurses should advocate for appropriate pain management strategies

\section{About the author}

Heather McLellan has been an emergency and critical care transport nurse for more than 40 years. She currently works as an Associate Professor with the Advanced Studies in Critical Care Nursing (ACCN) program at Mount Royal University and a bedside clinical nurse in the Foothills Medical Centre emergency department. Her research interests include hypothermia in transport and scholarship of teaching and learning related to nursing in highacuity practice areas.

\section{REFERENCES}

Abouzida, S., Bourgault, P., \& Lafrenaye, S. (2020). Observation of emergency room nurses managing pediatric pain: Care to be given... Care given. Pain Management Nursing, 21(6), 488-494. https://doi.org/10.1016/j.pmn.2020.03.002

Kahsay, D. T., \& Pitkajarvi, M. (2019). Emergency nurses' knowledge, attitude and perceived barriers regarding pain management in resource-limited settings: Cross-sectional study. BMC Nursing, 18(1), 56-56. https://doi.org/10.1186/ s12912-019-0380-9

Moceri, J. T., \& Drevdahl, D. J. (2014). Nurses' knowledge and attitudes toward pain in the emergency department. Journal of Emergency Nursing, 40(1), 6-12. https://doi.org/10.1016/j.jen.2012.04.014
Punches, B. E., Soliman, S., Freiermuth, C. E., Lane, B. H., \& Lyons, M. S. (2020). Emergency nurse perceptions of naloxone distribution in the emergency department. Journal of Emergency Nursing, 46(5), 675-681. https://doi.org/10.1016/j.jen.2020.05.006

Sardo, S., Galletta, M., Coni, E., Gonzalez, C. I. A., Piras, I., Pia, G., Evangelista, M., Musu, M., \& Finco, G. (2020). Nurses' behavior regarding pain treatment in an emergency department: A singlecenter observational study. Journal of Pain Research, 13, 23552359. https://doi.org/10.2147/JPR.S266087

World Health Organization (2021, August). Opioid overdose. https:// www.who.int/news-room/fact-sheets/detail/opioid-overdose

Look for supplemental materials such as author interviews and podcasts at www.CJEN.ca

The Canadian Journal of Emergency Nursing (CJEN) is the Official Journal of the National Emergency Nurses Association (NENA) of Canada. This article has been made available at no cost in partnership with NENA and the University of Alberta Libraries. 